\title{
Primary Sclerosing Cholangitis Complicated with Aphthoid Colitis Difficult to Diagnose from IgG4-Related Sclerosing Cholangitis
}

Jin Imai a, b Hitoshi Ichikawa ${ }^{b, c}$ Motoki Kaneko ${ }^{a, b}$

Hiroyuki Ito $^{b}$ Shinji Takashimizu ${ }^{b}$ Takayuki Shirai $^{b}$

Takuma Tajirid $^{d}$ Norihito Watanabe ${ }^{b}$ Hidekazu Suzuki ${ }^{a}$

aDepartment of Gastroenterology, Tokai University School of Medicine, Kanagawa, Japan; ${ }^{b}$ Department of Gastroenterology, Tokai University School of Medicine Hachioji Hospital, Tokyo, Japan; 'Center for Preventive Medicine, Keio University School of Medicine, Tokyo, Japan; ${ }^{\mathrm{d} D e p a r t m e n t}$ of Pathology, Tokai University School of Medicine Hachioji Hospital, Tokyo, Japan

Keywords

Primary sclerosing cholangitis · Aphtoid colitis · IgG4-related sclerosing cholangitis

\section{Abstract}

A 19-year-old man visited our hospital because of diarrhea and blood and liver dysfunction. Magnetic and endoscopic retrograde cholangiography revealed diffuse narrowing of the common biliary tract and intrahepatic biliary tract. A biopsy specimen from the narrowed area of the common biliary tract revealed IgG4-related sclerosing cholangitis (IgG4-SC). Colonoscopy showed multiple aphthoid colitis. He was treated with corticosteroids, and the levels of hepatobiliary enzymes, IgG, and IgG4 gradually decreased. This is an extremely rare case of colitis in which the patient had a phenotype of sclerosing cholangitis similar to IgG4-SC, which was finally diagnosed as primary sclerosing cholangitis. 
Imai et al.: PSC Complicated with Colitis Difficult to Diagnose from IgG4-SC

\section{Introduction}

Sclerosing cholangitis (SC) is defined as a condition with progressive stenosis of the bile ducts due to diffuse inflammation and fibrosis. Primary sclerosing cholangitis (PSC) is of unknown etiology, whereas secondary SC is caused by a variety of pathological conditions, including IgG4-related sclerosing cholangitis (IgG4-SC), which has been established as a clinical phenotype of systemic IgG4-related disease (IgG4-RD) found in the biliary tree [1]. Although the presentation of IgG4-SC and PSC are similar, the comorbidities, treatment response, and outcomes differ significantly; therefore, it is strongly advisable to be familiar with these two diseases to make the correct diagnosis [1]. However, diagnosis of these two diseases is sometimes difficult. Herein, we present a unique SC patient with a phenotype similar to that of IgG4-SC, who was finally diagnosed with PSC.

\section{Case Report}

A 19-year-old Japanese man with elevated serum levels of hepatobiliary enzymes was admitted to our hospital. Nine months prior, the patient had visited another hospital because of bloody diarrhea and liver dysfunction. Colonoscopy at the previous hospital revealed multiple aphthae in the terminal ileum including the ileocecal valve and the entire colon (Fig. 1a-d). He was suspected of having inflammatory bowel disease (IBD) and started taking mesalazine and ursodeoxycholic acid for liver dysfunction. Although his digestive symptoms resolved, the elevated serum levels of hepatobiliary enzymes still kept gradually increasing. He was then recommended to undergo a detailed examination at our hospital.

Physical examination at the time of admission revealed no abdominal findings or jaundice. He had no specific problems of past medical history and family history. Blood tests showed serum white blood cell count, 4,800/ $\mu \mathrm{L}$ (eosinophils, 360/ $\mu \mathrm{L}$ ); hemoglobin levels, 13.7/ $\mu \mathrm{L}$; amylase levels, $65 \mathrm{U} / \mathrm{L}$; total bilirubin level, $2.6 \mathrm{mg} / \mathrm{dL} ; \mathrm{AST}, 173 \mathrm{IU} / \mathrm{L}$; ALT, 219 IU/L; ALP; 2556 IU/L; $\gamma$-GTP, 567 IU/L; IgG levels, 2,421 mg/dL (normal range, $870-1,700 \mathrm{mg} / \mathrm{dL}$ ); and IgG4 levels, $248 \mathrm{mg} / \mathrm{dL}$ (normal range, $4.8-105 \mathrm{mg} / \mathrm{dL}$ ). Further tests including antinuclear antibodies, antimitochondrial antibodies, hepatitis B, hepatitis $\mathrm{C}$, and tumor markers were negative. Abdominal ultrasonography revealed the thickness of the common bile duct (Fig. 1e), and magnetic resonance cholangiopancreatography (MRCP) showed irregular contours of the intrahepatic duct (pruned-tree appearance) (Fig. 1f), which raised suspicion of PSC. Endoscopic retrograde pancreatocholangiography (ERCP) showed irregular narrowing of the common bile duct and intrahepatic duct, with no narrowing of the main pancreatic duct, and intraductal ultrasonography (IDUS) revealed wall thickening of the common bile duct (Fig. 1g). Histopathological examination of the common bile duct which was collected the transpapillary biopsy specimens under ERCP revealed moderate to severe lymphoplasmacytic infiltration with fibrosis. Immunostaining showed a large number of IgG4-positive plasma cells (more than 30 cells/highpower field) (Fig. 2), indicating the characteristic findings of IgG4-SC. However, considering his episode of bloody diarrhea, the presence of multiple aphthae in the whole colon, and the bile duct images from MRCP and ERCP, a diagnosis of PSC associated with undetermined colitis was suspected. For the treatment of liver dysfunction, he was started on treatment with $20 \mathrm{mg}$ corticosteroids $(0.5 \mathrm{mg} / \mathrm{kg} /$ day $)$, and the levels of hepatobiliary enzymes, IgG, and IgG4 gradually decreased (Fig. 3a). MRCP showed that the slightly dilated bile ducts were demonstrated mainly in the left intrahepatic bile duct with some narrowing (Fig. 3b).

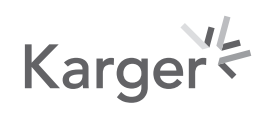



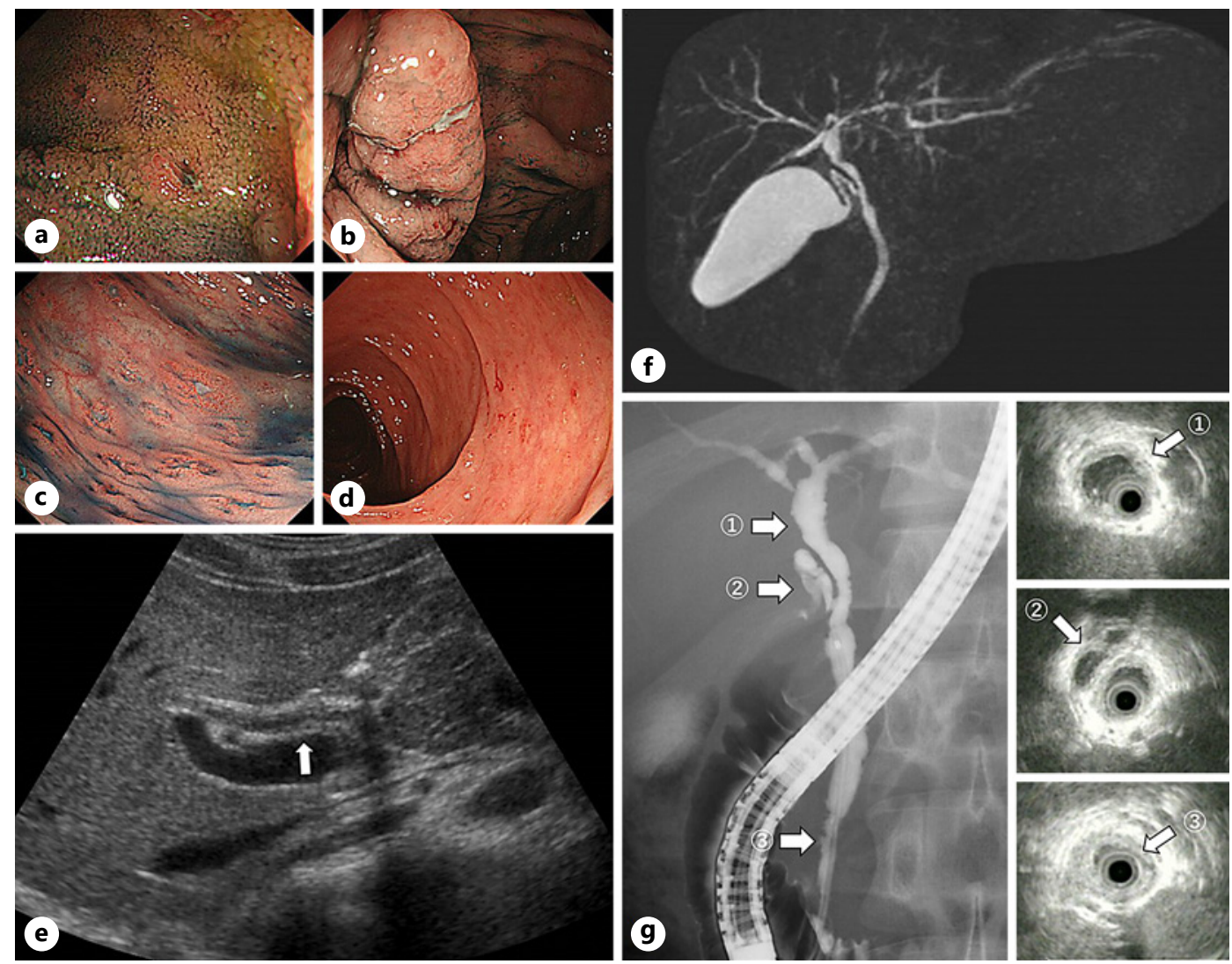

Fig. 1. The colonoscopy showed multiple aphthae in the terminal ileum (a) including the ileocecal valve (b) and the whole colon $(\mathbf{c}-\mathbf{d})$ at the previous doctor. $\mathbf{e}$ The abdominal ultrasound revealed the thickness of the common bile duct. $\mathbf{f}$ MRCP showed irregular contours of the intrahepatic duct, like a pruned-tree appearance. $\mathbf{g}$ ERCP showed irregular narrowing of the common bile duct and intrahepatic duct, and IDUS showed the wall thickness of the common bile duct.
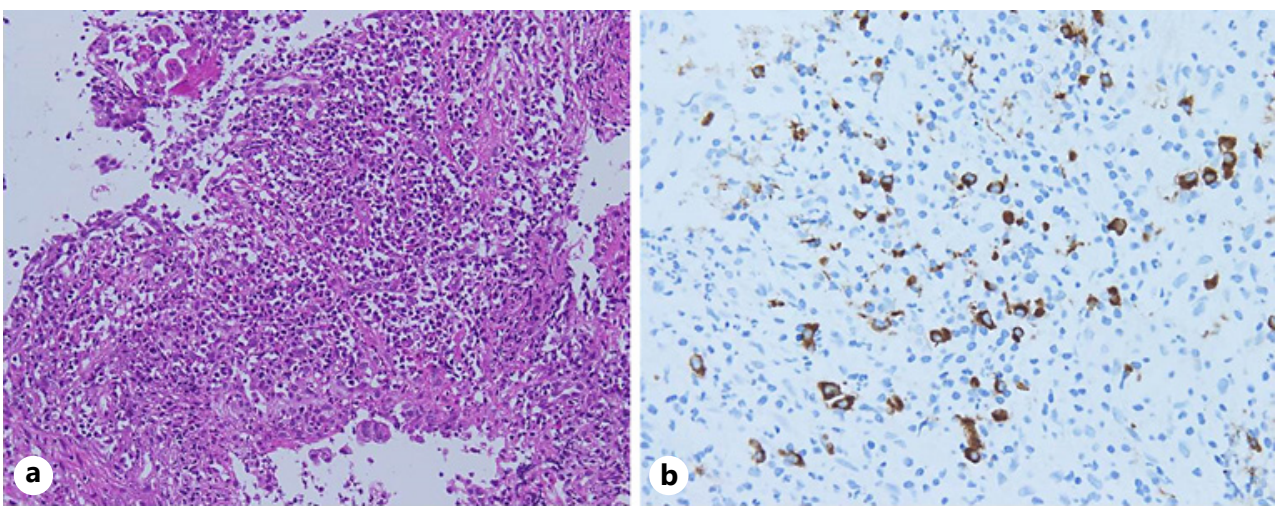

Fig. 2. a Histopathological examination of the common bile duct revealed moderate to severe lymphoplasmacytic infiltration with fibrosis (H\&E stain. $\times 400$ ) (b) Immunostaining study showed a large number of IgG4-positive plasma cells (more than 30 cells/high-power field) (IgG4 stain. $\times 400)$. 
Fig. 3. a He was started on treatment with $20 \mathrm{mg}$ corticosteroids, and the levels of hepatobiliary enzymes, IgG, and IgG4 gradually decreased. b MRCP showed that the slightly dilated bile ducts were demonstrated mainly in the left intrahepatic bile duct with some narrowing.

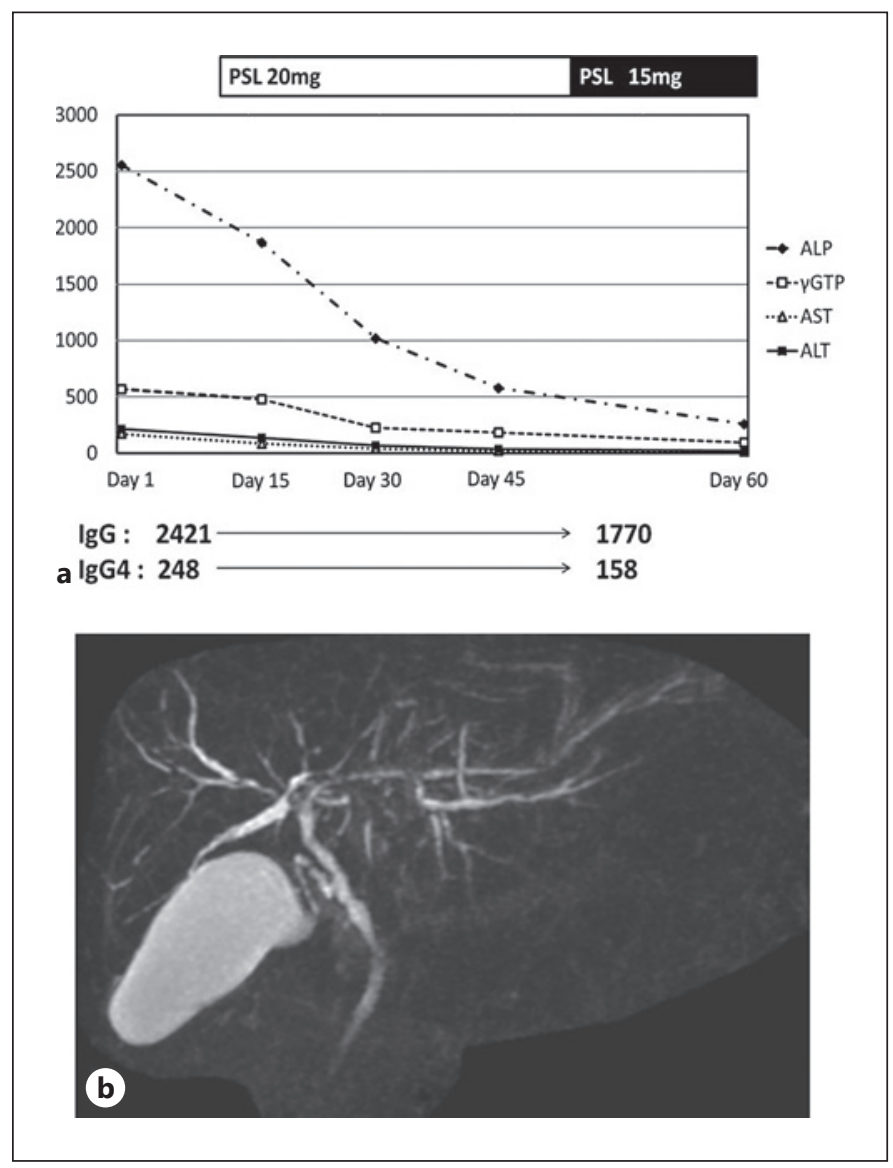

\section{Discussion}

Here, we report a rare case of PSC. We noted three important findings in this case.

First, this patient showed high serum IgG4 concentration and infiltration of abundant IgG4-positive plasma cells into the bile duct. Autoimmune pancreatitis (AIP) is a unique form of chronic pancreatitis characterized by a high serum IgG4 concentration and complications that include various extra-pancreatic manifestations, one of which is sclerosing cholangitis [2]. In this case, the abdominal ultrasound and abdominal CT scan did not show inflammatory swelling of the whole pancreas, and ERCP did not show irregular and narrow strictures of the main pancreatic duct, which is characteristic of type 1 AIP [3]. IgG4-SC is one of the most common extra-pancreatic manifestations of type 1 AIP; therefore, IgG4-SC often increased serum IgG4 levels (74\%), and abundant IgG4-positive cells are found in bile duct biopsy specimens (88\%) [4]. On the other hand, PSC shows lower serum IgG4 levels (9\%) [5] or local infiltration of IgG4-positive cells [6]. These findings may be difficult to distinguish between PSC and IgG4-SC. As the IDUS revealed the wall thickening of the lower common bile duct in this case, so we collected the transpapillary biopsy specimens from these lesions under ERCP. Naitoh et al. [7] reported that both IDUS and transpapillary biopsy after ERCP can provide further information rather than only biopsy in the diagnosis of IgG4-SC.

Second, this case showed symptomatic multiple aphthoid colitis that resembled IBD before taking mesalazine. The relationship between IBD and hepatobiliary disease is well recognized, especially in PSC [8]. The prevalence of IBD associated with PSC is as high as 
Table 1. Case comparison of PSC that responded to steroids

\begin{tabular}{llllll}
\hline & Age/sex & $\begin{array}{l}\text { AST/ALT/ALP, } \\
\text { IU/L }\end{array}$ & $\begin{array}{l}\text { Serum IgG4 } \\
\text { (over 135 mg/dL) }\end{array}$ & Colitis & $\begin{array}{l}\text { Pancreatic } \\
\text { involvement }\end{array}$ \\
\hline This case & 19/male & $173 / 219 / 2,556$ & + & Aphtoid colitis & - \\
Case 1 & $44 /$ male & $129 / 58 / 1,420$ & + & Indeterminate colitis & - \\
Case 2 & 29/male & $135 / 103 / 911$ & - & Pan-ulcerative colitis & - \\
Case 3 & 18/female & $141 / 174 / 569$ & - & Indeterminate colitis & - \\
\hline
\end{tabular}

$60 \%-80 \%$ in Western countries [9], although in Japan, IBD is found in only $21 \%-37 \%$ of PSC cases [10]. However, no association between IBD and IgG4-SC has been reported [11]. Only one study reported that $6 \%$ of IgG4-SC cases were associated with IBD in a Western country [12]. As such, an association with IBD is a very useful finding for the diagnosis of PSC. These findings supported the diagnosis of PSC in the present case.

Finally, the differential diagnosis of PSC and IgG4-SC is important because patients with IgG4-SC show a good response to steroid therapy [13]. In a systematic review of pediatric IgG4-related disease, the doses of prednisone were usually between 0.5 and $2 \mathrm{mg} / \mathrm{kg} / \mathrm{day}$ [14]. The present patient showed effective response to steroid therapy $(0.5 \mathrm{mg} / \mathrm{kg} /$ day). Therefore, this point is most difficult differentiation between PSC and IgG4-SC. However, Chen et al. [15] reported three PSC cases with IBD with a documented response to steroids. Compare to these 3 patients (Table.1), this case is similar in that it is young, has enteritis, and is not associated with pancreatic involvement. These findings suggested that there is sometimes no clear diagnosis between PSC and IgG4-SC; however, we must always consider PSC with poor prognosis in these situations [16]; therefore, we finally diagnosed the patient with PSC in this case. Of course, the possibility of IgG4-SC has not been completely denied, so it is necessary to carefully monitor the progress as long term. Further studies are necessary to elucidate these diseases.

\section{Acknowledgments}

This manuscript does not include any nonauthor contributors to acknowledge.

\section{Statement of Ethics}

The following research complies with the guidelines for human studies and was conducted ethically in accordance with the World Medical Association Declaration of Helsinki. The patient provided informed written consent to publish their case including the publication of the images. This study protocol was reviewed and the need for approval was waived by the Medical Ethics Committee on Human Research of Tokai University School of Medicine.

\section{Conflict of Interest}

The authors of this manuscript do not have any conflict of interest to declare.

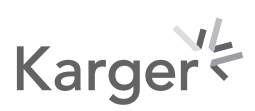


Imai et al.: PSC Complicated with Colitis Difficult to Diagnose from IgG4-SC

\section{Funding Sources}

This work was supported by Japan Society for the Promotion of Science (19K17413 to J.I.), Takeda Japan Medical Office Funded Research Grant 2019 (J.I.).

\section{Author Contributions}

J. Imai, H. Ichikawa, M. Kaneko, H. Ito, S. Takashimizu, T. Sirai, T. Tajiri, and N. Watanabe were responsible for the study concept and design, data collection, discussion, and drafting of the manuscript. J. Imai and H. Ichikawa reassessed the contents and English grammar of the manuscript. H. Suzuki supervised the whole process of the study. All the authors read and approved the final manuscript.

\section{Data Availability Statement}

All data generated or analyzed during this study are included in this article. Further inquiries can be directed to the corresponding author.

\section{References}

1 Tanaka A. IgG4-related sclerosing cholangitis and primary sclerosing cholangitis. Gut Liver. 2019;13(3):300-7.

2 Nishimori I, Otsuki M. Autoimmune pancreatitis and IgG4-associated sclerosing cholangitis. Best Pract Res Clin Gastroenterol. 2009;23(1):11-23.

3 Horiuchi A, Kawa S, Hamano H, Hayama M, Ota H, Kiyosawa K. ERCP features in 27 patients with autoimmune pancreatitis. Gastrointest Endosc. 2002;55(4):494-9.

4 Oseini AM, Chaiteerakij R, Shire AM, Ghazale A, Kaiya J, Moser CD, et al. Utility of serum immunoglobulin G4 in distinguishing immunoglobulin G4-associated cholangitis from cholangiocarcinoma. Hepatology. 2011;54(3):940-8.

5 Björnsson E, Chari S, Silveira M, Gossard A, Takahashi N, Smyrk T, et al. Primary sclerosing cholangitis associated with elevated immunoglobulin G4: clinical characteristics and response to therapy. Am J Ther. 2011; 18(3):198-205.

6 Nishino T, Oyama H, Hashimoto E, Toki F, Oi I, Kobayashi M, et al. Clinicopathological differentiation between sclerosing cholangitis with autoimmune pancreatitis and primary sclerosing cholangitis. J Gastroenterol. 2007;42(7):550-9.

7 Naitoh I, Nakazawa T, Ohara H, Ando T, Hayashi K, Tanaka H, et al. Endoscopic transpapillary intraductal ultrasonography and biopsy in the diagnosis of IgG4-related sclerosing cholangitis. J Gastroenterol. 2009;44(11): 1147-55.

8 Nakazawa T, Naitoh I, Hayashi K, Sano H, Miyabe K, Shimizu S, et al. Inflammatory bowel disease of primary sclerosing cholangitis: a distinct entity? World J Gastroenterol. 2014;20(12):3245-54.

9 Eaton JE, Talwalkar JA, Lazaridis KN, Gores GJ, Lindor KD. Pathogenesis of primary sclerosing cholangitis and advances in diagnosis and management. Gastroenterology. 2013;145(3):521-36.

10 Takikawa H, Takamori Y, Tanaka A, Kurihara H, Nakanuma Y. Analysis of 388 cases of primary sclerosing cholangitis in Japan: presence of a subgroup without pancreatic involvement in older patients. Hepatol Res. 2004; 29(3):153-9.

11 Zen Y, Harada K, Sasaki M, Sato Y, Tsuneyama K, Haratake J, et al. IgG4-related sclerosing cholangitis with and without hepatic inflammatory pseudotumor, and sclerosing pancreatitis-associated sclerosing cholangitis: do they belong to a spectrum of sclerosing pancreatitis? Am J Surg Pathol. 2004;28(9):1193-203.

12 Ghazale A, Chari ST, Zhang L, Smyrk TC, Takahashi N, Levy MJ, et al. Immunoglobulin G4-associated cholangitis: clinical profile and response to therapy. Gastroenterology. 2008;134(3):706-15.

13 Nakazawa T, Ohara H, Sano H, Ando T, Aoki S, Kobayashi S, et al. Clinical differences between primary sclerosing cholangitis and sclerosing cholangitis with autoimmune pancreatitis. Pancreas. 2005;30(1):20-5.

14 Karim F, Loeffen J, Bramer W, Westenberg L, Verdijk R, van Hagen M, et al. IgG4-related disease: a systematic review of this unrecognized disease in pediatrics. Pediatr Rheumatol Online J. 2016;14(1):18.

15 Chen JH, Deshpande V. Inflammatory nodules identify steroid-responsive primary sclerosing cholangitis. Int J Surg Pathol. 2018;26(5):402-9.

16 Martin EF, Levy C. Timing, management, and outcomes of liver transplantation in primary sclerosing cholangitis. Semin Liver Dis. 2017;37(4):305-13. 